Received: 2018.04.14 Accepted: 2018.07.11 Published: 2018.10.11

\title{
Hepatic Involvement in Systemic Sarcoidosis
}

Authors' Contribution:

Study Design A

Data Collection B

Statistical Analysis C

Data Interpretation D

Manuscript Preparation

Literature Search F

Funds Collection $G$

\author{
ABCDEFG 1 Abdisamad M. Ibrahim \\ BDEFG 1 Bishal Bhandari \\ ACDEFG 1 Paolo K. Soriano \\ ABCDFG 2 Zafar Quader \\ BDEG 3 John Z. Gao \\ ACDEFG 4 Dmitry Shuster \\ AEFG 5 Chaitanya K. Mamillapalli
}

1 Department of Internal Medicine, Southern Illinois University School of Medicine, Springfield, IL, U.S.A.

2 Department of Gastroenterology and Hepatology, Southern Illinois University School of Medicine, Springfield, IL, U.S.A.

3 Pathology Associates of Central Illinois, Memorial Medical Center, Springfield, IL, U.S.A.

4 Department of Gastroenterology and Hepatology, Springfield Clinic, Springfield, IL, U.S.A.

5 Department of Endocrinology, Springfield Clinic, Springfield, IL, U.S.A.
Corresponding Author: Conflict of interest:
Abdisamad M. Ibrahim, e-mail: qalbinoor@gmail.com

None declared

\begin{abstract}
Patient: $\quad$ Female, 68
Final Diagnosis: Hepatic sarcoidosis

Symptoms: Abdominal pain • nausea • weakness

Medication:

Clinical Procedure: Liver biopsy

Specialty: Gastroenterology and Hepatology

Objective: Rare disease

Background: Sarcoidosis is a systemic disease that can affect any organ, including the liver. It is manifested by the presence of non-caseating granulomas within involved organs, most commonly the pulmonary, lymphatic, and hepatic system. Unlike pulmonary or lymphatic involvement, hepatic involvement is usually asymptomatic and it is underdiagnosed. Here, we report a case of a patient with a history of pulmonary sarcoidosis who developed hepatic sarcoidosis.

Case Report: 68-year-old female with pulmonary sarcoidosis with a 2-week history of severe abdominal pain and epigastric tenderness presented to our center. Abdominal magnetic resonance imaging (MRI) demonstrated mild hepatic fibrosis and cirrhosis. A thorough workup was performed including a liver biopsy which showed chronic non-necrotizing granulomas consistent with sarcoidosis. She was started on prednisone and subsequently improved. The patient was symptom-free on follow-up 1 month later.

Conclusions: The majority of patients with hepatic sarcoidosis are usually asymptomatic, with only 5-30\% presenting with abdominal pain, jaundice, nausea, vomiting, and hepatosplenomegaly. In rare cases, hepatic sarcoidosis can lead to cholestasis, portal hypertension, cirrhosis, or Budd-Chiari syndrome. Treatment with steroids is the mainstay of therapy; however, in severe cases, patients may require liver transplantation. This case report demonstrates that hepatic sarcoidosis is a serious condition, and if not treated, can lead to portal hypertension and cirrhosis. In patients with sarcoidosis, early detection and longitudinal follow-up is important in preventing overt liver failure.
\end{abstract}

MeSH Keywords: Cholestasis • Liver Cirrhosis • Sarcoidosis

Full-text PDF: https://www.amjcaserep.com/abstract/index/idArt/910600 


\section{Background}

The prevalence of sarcoidosis in the United States is estimated to be $1-40$ per 100000 people [1]. Hepatic involvement occurs in $11.5 \%$ of patients with sarcoidosis [2] and the prevalence is twice more likely in African Americans [3] Hepatic sarcoidosis is usually clinically silent, however, in severe cases, it can manifest as cholestasis, cirrhosis, portal hypertension, and ultimately, liver failure [1-3].

\section{Case Report}

A 68-year-old Caucasian female with pulmonary sarcoidosis, hypertension, and type 2 diabetes presented to the Emergency Department with severe abdominal pain. The pain was dull, constant, and began approximately 1 month prior with progression and exacerbation over the previous 2 weeks. The pain was located in her right upper quadrant and epigastric region without radiation, there was no correlation with food intake or change in position. Associated symptoms were weakness, dizziness, and nausea. She denied any systemic signs of infection including fevers, chills, vomiting, and diarrhea. There was no history of viral hepatitis, or alcohol or illicit drug use.
On physical examination, she was normotensive, tachycardic, breathing normally, and saturating well on room air but she appeared to be in moderate distress. Her respiratory examination was benign. Her cardiac examination as also benign except for a mild aortic systolic murmur. Her abdomen was soft and non-distended with hypoactive bowel sounds. She was tender to palpation on her epigastrium and right upper quadrant. Mild hepatomegaly was appreciated on deep palpation. The patient did not have a positive Murphy's sign but did have slight voluntary guarding without rebound tenderness.

Admission lab work demonstrated a cholestatic pattern of liver injury. Other significant labs included, elevated calcium level and 1,25-hydroxy vitamin D (Table 1). A magnetic resonance imaging (MRI) of the liver demonstrated mild fibrosis and cirrhosis of the liver with hepatosplenomegaly (Figure $1 \mathrm{~A}$ ). A computed tomography (CT) of the chest and abdomen showed scattered calcified granulomas within the lungs, liver, and spleen (Figure 1B). The pancreas was normal and there were some enlarged upper abdominal lymph nodes. The patient underwent a thorough workup for etiology of granulomatous liver disease and pertinent negative labs included: anti-mitochondrial antibody, anti-smooth antibody, antinuclear antibody, and viral hepatitis serology. Finally, a liver biopsy showed chronic

Table 1. Laboratory evaluation results and reference range.

\begin{tabular}{|c|c|c|}
\hline Test & Result & Reference range \\
\hline Aspartate aminotransferase & $23 \mathrm{IU} / \mathrm{L}$ & $(0-41 \mathrm{IU} / \mathrm{L})$ \\
\hline Alanine aminotransferase & $11 \mathrm{IU} / \mathrm{L}$ & $(0-45 \mathrm{IU} / \mathrm{L}$ \\
\hline Alkaline phosphatase & $512 \mathrm{IU} / \mathrm{L}$ & $(30-130$ IU/L) \\
\hline Total bilirubin & $1.4 \mathrm{mg} / \mathrm{dL}$ & $(0-1.0 \mathrm{mg} / \mathrm{dL})$ \\
\hline $\mathrm{PT} / \mathrm{INR}$ & $14.4 \mathrm{sec} / 1.1$ & $(12.5-14.7 \mathrm{sec} / 0.9-1.1)$ \\
\hline Albumin & $2.5 \mathrm{~g} / \mathrm{dL}$ & $(3.5-5.5 \mathrm{~g} / \mathrm{dL})$ \\
\hline Serum calcium & $15.5 \mathrm{mg} / \mathrm{dL}$ & $(8.5-10.5 \mathrm{mg} / \mathrm{dL})$ \\
\hline Intact PTH & $3 \mathrm{pg} / \mathrm{mL}$ & $(12-88 \mathrm{pg} / \mathrm{mL})$ \\
\hline PTHrP & $2 \mathrm{pmol} / \mathrm{L}$ & $(0-3.4 \mathrm{pmol} / \mathrm{L})$ \\
\hline Total 25 hydroxy vitamin D2 D3 & $24 \mathrm{ng} / \mathrm{mL}$ & $(30-100 \mathrm{ng} / \mathrm{mL})$ \\
\hline 1,25-hydroxy vitamin D & $151 \mathrm{pg} / \mathrm{mL}$ & $(19.9-79.3 \mathrm{pg} / \mathrm{mL})$ \\
\hline $\mathrm{ACE}$ & $<5 \mathrm{U} / \mathrm{L}$ & $(9-67$ IU/L) \\
\hline Anti-mitochondrial antibody & Negative & \\
\hline Anti-smooth antibody & Negative & \\
\hline Antinuclear antibody & Negative & \\
\hline Viral hepatitis serology & Negative & \\
\hline \multicolumn{3}{|c|}{ Additional tests on liver biopsy } \\
\hline Fungal cultures & Negative & \\
\hline Acid-fast bacilli & Negative & \\
\hline Gram stain and cultures & Negative & \\
\hline
\end{tabular}



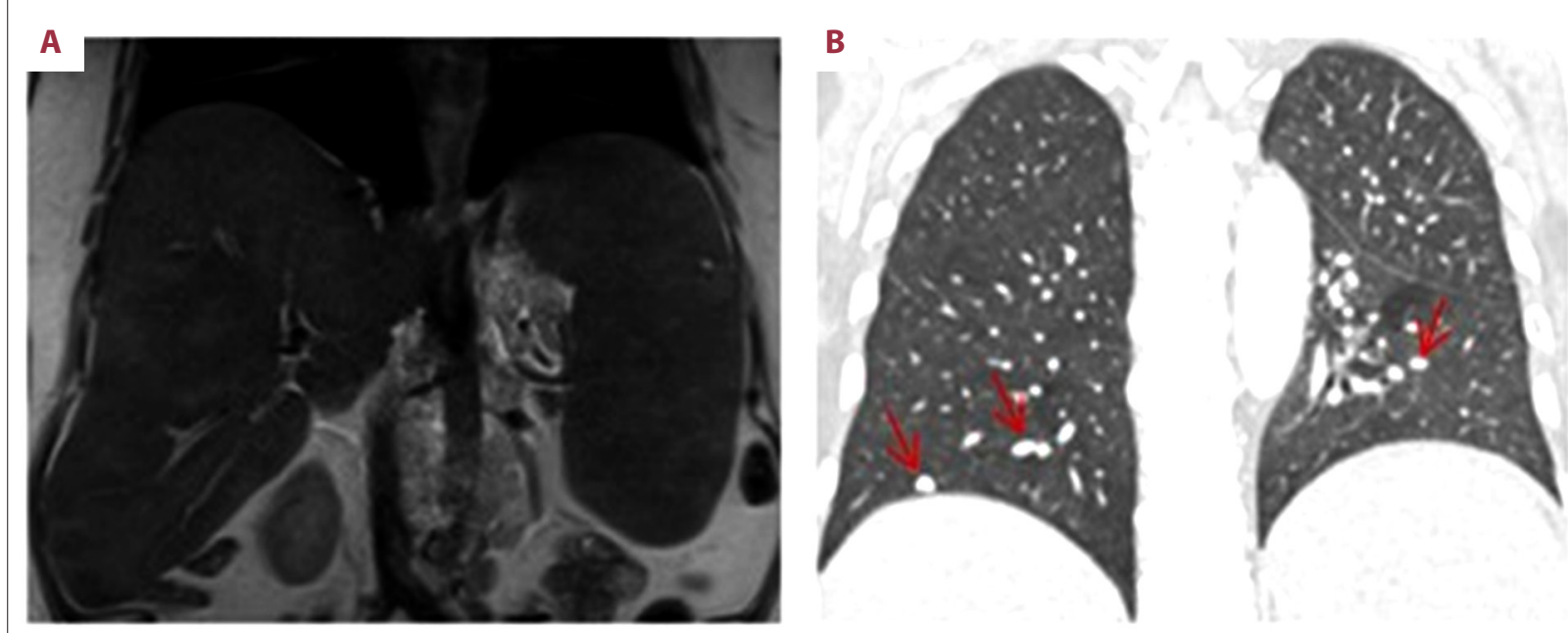

Figure 1. (A) Coronal view of abdominal magnetic resonance imaging (MRI). MRI demonstrates mild fibrosis and cirrhosis in the liver. Hepatosplenomegaly is appreciated on this image as well. (B) Chest computed tomography (CT) with contrast. CT chest demonstrates calcified granulomas within the lungs.
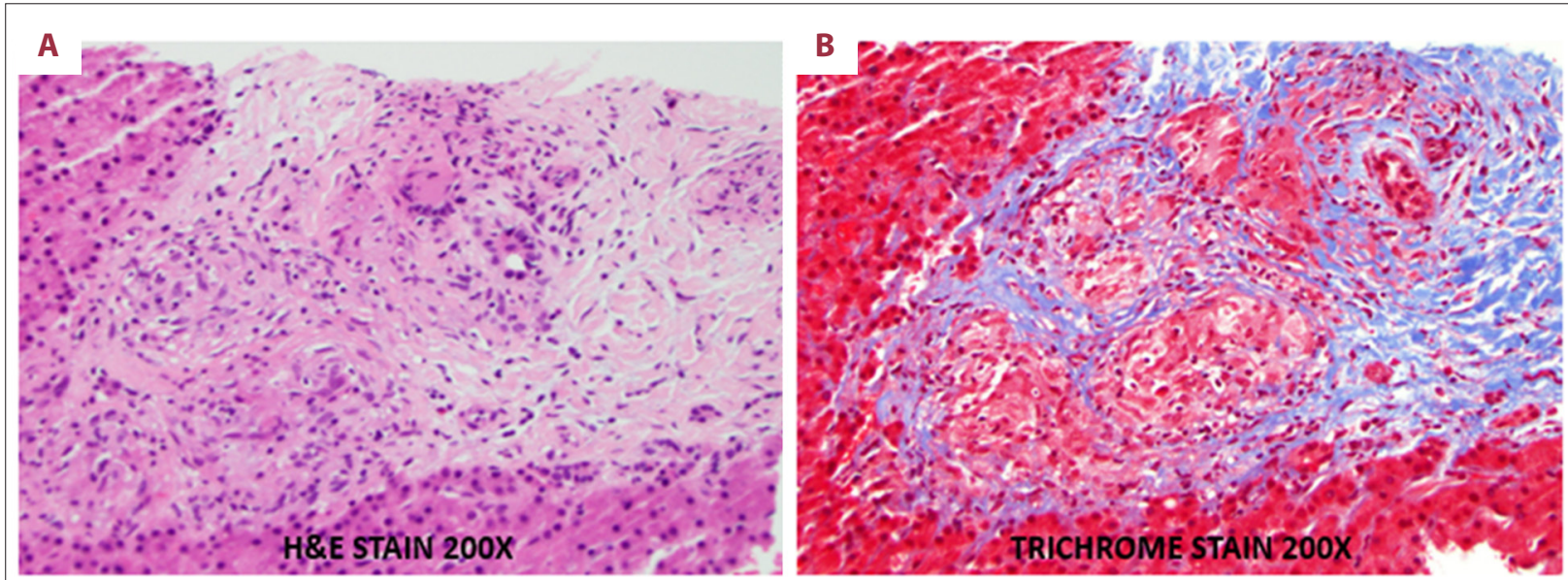

Figure 2. Histopathology. (A) hematoxylin and eosin section showing chronic granulomatous hepatitis with compact non-necrotizing granulomas. Notice the absence stainable iron in the hepatocytes with the trichrome stain (B).

granulomatous hepatitis with compact non-necrotizing granulomas, minimal steatosis, and no stainable iron seen in hepatocytes (Figure 2A, hematoxylin and eosin (H\&E stain), 200x, and Figure 2B, trichome stain, 200x). Fungal cultures, acid-fast bacilli, and gram stain were all negative on biopsy. Cardiac MRI was negative for cardiac involvement of her sarcoidosis. Esophagogastroduodenoscopy was performed showing small gastric sessile polyps but otherwise negative for varices.

Elevated serum calcium, low intact parathyroid hormone (PTH), and elevated 1,25 hydroxy vitamin D were consistent with hypercalcemia secondary to sarcoidosis. She had a known history of pulmonary sarcoidosis; however, the hepatic involvement made this case noteworthy. Our differential diagnosis for non-caseating granuloma included hepatic sarcoidosis, fungal infections, tuberculosis, primary biliary cirrhosis, lymphoma, and drug reactions. She tested negative for anti-mitochondrial antibody, fungal infections, tuberculosis, and lymphoma. The patient was not on any medications that have been known to cause non-caseating granulomas, thus raising suspicion for hepatic sarcoidosis, which was ultimately confirmed via histology.

The patient was treated with prednisone $30 \mathrm{mg}$ twice a day in the hospital and was discharged on a prolonged prednisone course. Her symptoms improved, and she was discharged home after an 11-day hospital stay. The patient did not have reoccurrence of her symptoms at a follow-up 1 month later. 


\section{Discussion}

The majority of patients with hepatic sarcoidosis are asymptomatic, with only $5-30 \%$ presenting with abdominal pain, jaundice, nausea, vomiting, and hepatosplenomegaly [1]. In rare cases, hepatic sarcoidosis can lead to cholestasis, portal hypertension, cirrhosis, or Budd-Chiari syndrome [3].

Patients with hepatic sarcoidosis seldom have elevated transaminases and alkaline phosphatase. Hepatic and splenic lesions are seen in $5 \%$ and $15 \%$ of patients, respectively [3]. Our patient had radiographic evidence of hepatic cirrhosis and portal hypertension but was clinically compensated. Previous studies have failed to prove the correlation of transaminases with disease severity. In those with severe hepatic involvement, higher levels of both transaminases and alkaline phosphatase might be seen [3]. In addition, the patient's esophagogastroduodenoscopy did not show evidence of varices. Hypoalbuminemia and hyperbilirubinemia are rarely seen and are associated with cirrhosis [4].

Liver biopsy is recommended for patients suspected of having symptomatic hepatic sarcoidosis (e.g., elevated serum aminotransferase and alkaline phosphatase) [5]. Other diseases that can cause hepatic granulomas, such as fungal infections, tuberculosis, primary biliary cirrhosis, lymphoma, and drug reactions, should be excluded. In symptomatic patients, an increase in serum angiotensin converting enzyme level might be seen, but levels are normal in $25 \%$ of untreated patients [6].

Hypercalcemia occurs in $11 \%$ of patients with sarcoidosis, while hypercalciuria and renal calculi occur in $40 \%$ and $10 \%$, respectively [4]. Granulomas contain 25-hydroxyvitamin D-1 $\alpha$ hydroxylase, which converts 25 -hydroxyvitamin $D$ to 1,25 dihydroxyvitamin D, thus leading to hypercalcemia. Early recognition and treatment of hypercalcemia is important before intrarenal calcium deposition occurs, which leads to renal failure [4].

\section{References:}

1. Tan CB, Rashid S, Rajan D et al: Hepatic sarcoidosis presenting as portal hypertension and liver cirrhosis: Case report and review of the literature. Case Rep Gastroenterol, 2012; 6(1): 183-89

2. Ayyala US, Padilla ML: Diagnosis and treatment of hepatic sarcoidosis. Curr Treat Options Gastroenterol, 2006; 9(6): 475-83

3. Tadros M, Forouhar F, Wu GY: Hepatic sarcoidosis. J Clin Transl Hepatol, 2013; 1(2): 87-93
In patients with symptomatic hepatic sarcoidosis, treatment with steroids is recommended for at least 6 months. Kennedy et al. reported on 63 patients with hepatic sarcoidosis when treated with steroids, out of which one-third had a complete clinical response, one-third had a partial response, and one-third had no response [7]. The definitive treatment of symptomatic hepatic sarcoidosis is liver transplantation; however, recurrence of the disease is possible in the new organ [7].

\section{Conclusions}

Hepatic sarcoidosis should be considered in patients with underlying systemic sarcoidosis presenting with abdominal pain, jaundice, nausea, vomiting, and hepatosplenomegaly. Prior to liver biopsy, other etiologies that may result in hepatic granulomas should first be excluded. Treatment with steroids is the mainstay of therapy for hepatic sarcoidosis, however, in severe cases patients may require liver transplantation. Our case demonstrates that hepatic sarcoidosis is a serious condition, and if not treated, could lead to a variety of complications, including portal hypertension, cirrhosis, or Budd-Chiari syndrome. In patients with sarcoidosis, early detection and longitudinal follow-up is important in preventing overt liver failure.

\section{Acknowledgement}

We thank Ayan Aden and Lydia Howes for proofreading the manuscript and organizing the references.

\section{Conflict of interest}

None.

4. Lannuzzi MC, Rybicki BA, Teirstein AS: Sarcoidosis. N Engl J Med, 2007; 357 2153-65

5. Cremers J, Drent M, Driessen A et al: Liver-test abnormalities in sarcoidosis. Eur J Gastroenterol Hepatol, 2012; 24(1): 17-24

6. Studdy PR, Bird R: Serum angiotensin converting enzyme in sarcoidosis its value in present clinical practice. Ann Clin Biochem, 1989; 26: 13-18

7. Kennedy PTF, Zakaria N, Modawi SB et al: Natural history of hepatic sarcoidosis and its response to treatment. Eur J Gastroenterol Hepatol, 2006 18(7): 721-26 